\title{
Comparative Treatment Patterns, Resource Utilization, and Costs in Stimulant-Treated Children with ADHD Who Require Subsequent Pharmacotherapy with Atypical Antipsychotics Versus Non-Antipsychotics
}

\author{
Vanja Sikirica, PharmD, MPH; Steven R. Pliszka, MD; Keith A. Betts, PhD; \\ Paul Hodgkins, PhD, MSc; Tom Samuelson, BA; Jipan Xie, MD, PhD; M. Haim Erder, PhD; \\ Ryan Dammerman, MD, PhD; Brigitte Robertson, MD; and Eric Q. Wu, PhD
}

\begin{abstract}
BACKGROUND: Although not indicated for attention-deficit/hyperactivity disorder (ADHD), atypical antipsychotics (AAPs) are commonly prescribed for children with ADHD. The treatment patterns, resource utilization, and costs associated with AAPs relative to non-antipsychotic medications have not been evaluated for children with ADHD.

OBJECTIVE: To compare treatment patterns, resource utilization, and costs to U.S. third party payers between stimulant-treated ADHD children who switch to or augment their stimulant treatment with AAPs (risperidone, aripiprazole, quetiapine, olanzapine, ziprasidone, paliperidone, and clozapine) compared with non-antipsychotic medications (atomoxetine, clonidine immediate-release (IR), guanfacine IR, dexmethylphenidate, mixed amphetamine salts, methylphenidate, lisdexamfetamine, and dextroamphetamine).
\end{abstract}

METHODS: Patients with at least one ADHD diagnosis (ICD-9-CM codes 314.00 or 314.01 ) and at least one stimulant medication claim between January 1, 2005 and December 31, 2009, were identified from a large U.S. commercial medical/pharmacy claims database. Patients were classified into the AAP cohort if they had a claim for an AAP following a stimulant fill or into the non-antipsychotic cohort if they had a claim for a nonantipsychotic medication after a stimulant fill and no AAP claims. The index date was defined as the date of the first fill of the AAP or a randomly selected eligible non-antipsychotic medication. Patients were eligible for inclusion if they were aged 6-12 as of the index date and had at least 18 months of continuous eligibility. Patients were excluded if they had a psychiatric diagnosis for which AAPs were approved by the U.S. Food and Drug Administration (FDA) or commonly used. Patients in the non-antipsychotic group were matched 1:1 to patients in the AAP group using a propensity score generated from a logistic regression that included demographics, treatments, resource utilization, and comorbidities during the 6 months prior to the index date. All outcomes were measured during the 12 months following the index date. Treatment patterns were compared using KaplanMeier (KM) estimates and Cox proportional hazards models. Annual resource utilization was compared using McNemar's test and Poisson regression. Costs were estimated from the perspective of U.S. third-party payers and were adjusted to $\mathbf{2 0 1 0}$ dollars using the medical component of the Consumer Price Index. Both all-cause and mental health-related costs were examined and compared using Wilcoxon signed-rank tests.

RESULTS: Of the 22,622 patients with ADHD identified to have used AAPs after a stimulant, 15,664 (69\%) patients did not have a psychiatric diagnosis for which AAPs were FDA-indicated or commonly used. Among the 84,558 patients using non-antipsychotics after a stimulant, 81,397 (96\%) did not have such psychiatric diagnoses. A total of 2,127 children in the AAP cohort and 16,508 children in the non-antipsychotic cohort met all of the study inclusion criteria. After propensity score matching, 1,857 children (358 switchers and 1,499 augmenters) were included in each of the matched cohorts. The baseline characteristics were well balanced between the matched cohorts. In the 12 months post-index date, children treated with AAPs were more likely to experience switching (KM: $17.2 \%$ vs. $10.4 \%$ at 12 months; $\mathrm{HR}=1.75$ ) and augmentation (KM: $43.4 \%$ vs. $22.4 \%$ at 12 months; $\mathrm{HR}=2.62$ ) than the non-antipsychotic group (both $P<0.001$ ). Rates of discontinuation were similar between groups (KM: $71.8 \%$ vs. $71.7 \%$ at 12 months; HR=0.98, $P=0.600)$. The AAP cohort also had higher mean numbers of hospitalizations, emergency room visits, and outpatient visits $(0.08$ vs. $0.03,0.34$ vs. $0.25,14.1$ vs. 12.7 per patient, respectively; event rate ratios $=2.61,1.33$, and 1.11 , respectively; all $P<0.001$ ). The AAP group also incurred higher all-cause mean medical, prescription drug, and total health care costs compared with the non-antipsychotic group $(\$ 3,090$ vs. $\$ 2,238$; $\$ 3,844$ vs. $\$ 2,509 ; \$ 6,934$ vs. $\$ 4,748$, respectively; all $P<0.001)$. Patients in the AAP group also incurred higher mean total, medical, and drug costs related to mental health ( $\$ 5,057$ vs. $\$ 2,859 ; \$ 1,555$ vs. $\$ 964 ; \$ 3,502$ vs. $\$ 1,895$, respectively; all $P<0.001$ ).

CONCLUSIONS: Stimulant-treated children with ADHD who switched to or augmented with AAPs versus non-antipsychotics had significantly greater rates of subsequent augmentation and health care resource utilization as well as higher total health care costs. Further research and/or drug utilization reviews may be warranted to fully evaluate the clinical and economic outcomes of pediatric ADHD patients who are receiving AAPs.

\section{J Manag Care Pharm. 2012;18(9):676-89}

Copyright $\odot 2012$, Academy of Managed Care Pharmacy. All rights reserved.

\section{What is already known about this subject}

- Atypical antipsychotics (AAPs) are one of the most commonly prescribed classes of medications in the United States. Use of AAPs for indications not approved by the U.S. Food and Drug Administrion (FDA) account for the majority of treatment with AAPs and has been growing faster than the use for FDA-approved indications

- Several studies have shown that AAPs, particularly risperidone, can reduce attention-deficit/hyperactivity disorder (ADHD) symptoms in patients with ADHD who have comorbid conditions, such as bipolar disorder or mental retardation. However, overall the strength of evidence is low, and AAPs are not approved for the treatment of ADHD.

- There is no published study in the peer-reviewed literature on the use of AAPs in ADHD and their impact on treatment patterns, resource utilization, and costs. 


\section{Comparative Treatment Patterns, Resource Utilization, and Costs in Stimulant-Treated Children with ADHD Who Require Subsequent Pharmacotherapy with Atypical Antipsychotics Versus Non-Antipsychotics}

\section{What this study adds}

- This study was the first to examine the treatment patterns and economic outcomes associated with AAP use in the treatment of ADHD using real-world data.

- After controlling for potential confounding variables, including comorbidities, children treated with AAPs had significantly greater subsequent drug augmentation; higher incidence of hospitalization, emergency room, and outpatient visits; and incurred higher all-cause medical, prescription drug, and total health care costs, as well as mental health-related costs compared with children treated with non-antipsychotic medications in the 12 months post-treatment initiation.

A ttention-deficit/hyperactivity disorder (ADHD) is the most commonly diagnosed neurobehavioral disorder of childhood. ${ }^{1}$ In the United States, the percentage of children aged 4 to 17 years with ADHD increased from $7.8 \%$ during 2003 to 9.5\% during 2007.2 If left untreated, ADHD may pose a significant barrier to personal development and cause a substantial psychological and financial burden to patients' families. ${ }^{3}$ The total economic burden, including direct and indirect costs, associated with ADHD in children and adolescents is estimated to be $\$ 42.5$ billion (in 2005 U.S. dollars) per year. ${ }^{4}$ Results of multiple cost studies consistently indicated that children with ADHD had higher annual medical costs than children without ADHD. 5-11

Treatment options for ADHD include medication management, behavioral treatment, or combination of the two, with medication management being the most cost-effective. ${ }^{12}$ Stimulants are an effective first-line treatment option for the majority of ADHD patients, with about $70 \%$ of patients responding to treatment in the short term (within 6-10 weeks) ${ }^{13,14}$ However, a subset of the patient population treated with stimulants will seek an alternative medication regimen for a variety of reasons, such as lack of improvement, tolerability issues, or the possible negative societal perception of stimulants. These patients may switch to another stimulant or augment therapy with a nonstimulant, but increasingly, they are turning to atypical antipsychotics (AAPs). ${ }^{15-17}$

With an annual sales of more than $\$ 13$ billion dollars, accounting for nearly $5 \%$ of all drug expenditures in the United States, AAPs are one of the most common and costly classes of prescription drugs. ${ }^{18,19}$ Use of AAPs for indications not approved by the U.S. Food and Drug Administration (FDA) accounts for the majority of treatment with AAPs. ${ }^{19}$ A recent study using data from the National Ambulatory Medical Care Survey and the National Hospital Ambulatory Medical Care Survey found that ADHD was the most common diagnosis associated with an antipsychotic prescription for children aged 2 to 18 years. ${ }^{15}$ A separate analysis of 11,700 Arkansas
Medicaid-covered children who were newly treated with AAPs also found the most common condition was ADHD followed by depression, conduct disorder, oppositional defiant disorder, and adjustment reactions. ${ }^{16}$ An exploratory study in a Canadian ADHD clinic found that nearly one in five children with ADHD was prescribed AAPs off-label to treat ADHD in 2009. ${ }^{17}$

The clinical use of AAPs in pediatric patients with ADHD alone is not fully justified due to the limited evidence from randomized control trials. ${ }^{20,21}$ Existing clinical trials of AAPs for ADHD had mixed findings in terms of efficacy and were conducted in children with severe comorbid conditions, such as bipolar disorder or mental retardation. ${ }^{22-25}$ In most of these studies, the AAP was targeted at the comorbid symptoms rather than ADHD itself. In 2004, a consensus of international experts on ADHD and disruptive behavior disorders (DBDs) suggested augmenting psychostimulant treatment with risperidone as a second-line treatment option for the DBD (i.e., if patients experience insufficient response at the maximum recommended psychostimulant dose) in children with ADHD and conduct disorders. ${ }^{26}$

To date, no peer-reviewed studies that examine the economic outcomes of using AAPs in ADHD patients have been published. Given the high prevalence of AAP use, payers and clinicians may want to understand the treatment patterns, resource use, and economic impact of AAP use to support informed decision making about the appropriate medications to treat ADHD patients who are not satisfied or whose symptoms are not adequately controlled with their current stimulant therapy. The objective of this study was to compare treatment patterns, resource utilization, and health care costs between stimulant-treated ADHD children who switched to or augmented their stimulant treatment with AAPs versus non-antipsychotic medications from a U.S. third-party payer perspective.

\section{Methods}

\section{Data}

This retrospective cohort study was conducted utilizing data from the Thomson Reuters MarketScan Commercial Claims $\&$ Encounters database (Thomson Reuters, Chicago, IL) for the period of January 1, 2005, to December 31, 2009. These data include commercial health insurance claims (inpatient and outpatient medical and outpatient pharmacy) and enrollment data from large employers and health plans across the United States. Such plans provide private health care coverage for more than 45 million employees, their spouses, and dependents. This administrative claims database includes a variety of fee-for-service, preferred provider organization, and capitated health plans.

\section{Sample Selection}

The study sample consisted of patients with at least one medical claim associated with a primary diagnosis of ADHD 


\section{Comparative Treatment Patterns, Resource Utilization, and Costs in Stimulant-Treated Children with ADHD Who Require Subsequent Pharmacotherapy with Atypical Antipsychotics Versus Non-Antipsychotics}

during the period of January 1, 2005, to December 31, 2009. Claims were identified by International Classification of Diseases, 9th Revision, Clinical Modification (ICD-9-CM) codes 314.00 (Attention-deficit disorder of childhood without hyperactivity) and 314.01 (Attention-deficit disorder of childhood with hyperactivity). Patients were further required to have had a pharmacy claim for a stimulant (dexmethylphenidate, mixed amphetamine salts, methylphenidate, lisdexamfetamine, or dextroamphetamine) during the period of January 1, 2005, to December 31, 2009, which were identified by mapping National Drug Codes (NDCs) provided in the MarketScan dataset to Generic Product Identifier codes (GPI; Medi-Span, Inc., Indianapolis, IN; Appendix). The date of the first stimulant claim was defined as the initial stimulant date.

Patients were classified into either the AAP cohort or the non-antipsychotic cohort based on the following criteria. Patients were selected into the AAP cohort if they had a pharmacy claim for risperidone, aripiprazole, quetiapine, olanzapine, ziprasidone, paliperidone, or clozapine, and the first fill for the AAP occurred any time following the initial stimulant date. Patients with pharmacy claims for AAPs prior to the initial stimulant date were excluded from the study. The date of the first AAP pharmacy claim was defined as the index date, and the AAP filled was defined as the index therapy. Patients with no pharmacy claims for AAPs and at least one pharmacy claim after the initial stimulant date for atomoxetine, guanfacine immediate-release (IR), clonidine IR, or a stimulant of a different class than the initial stimulant (Appendix) were selected into the non-antipsychotic cohort. For non-antipsychotic patients with pharmacy claims for more than one of the eligible medications as described above (e.g., having claims for both atomoxetine and guanfacine IR after the initial stimulant), the index therapy was randomly selected. Random selection was employed to match the inclusion criteria of the AAP cohort (which may have one or more non-antipsychotic medications prior to the index date) in an unbiased manner. The index date was defined as the date of the first fill for the index therapy.

In addition, patients in both cohorts were required to meet the following criteria: (a) Patients were aged 6 to 12 years as of the index date; (b) patients had at least 30 days of a stimulant supply before the index date; (c) patients had at least 18 months (6 months pre- and 12 months post-index date) of continuous eligibility; (d) patients did not have any medical claim associated with the following diagnoses during the 6 months preand 12 months post-index date: bipolar disorder (ICD-9-CM: 296.0, 296.1, 296.4-296.8); schizophrenia (ICD-9-CM: 295); psychotic disorder with delusions/hallucinations (ICD-9-CM: 293.81, 293.82); paranoia (ICD-9-CM: 297.1, 297.3); psychosis (ICD-9-CM: 298.8, 298.9); tics/Tourette's syndrome (ICD9-CM: 307.2, 307.23); or dementia (ICD-9-CM: 290, 294.1). These included conditions that can be identified through ICD-9 codes and are either approved by the FDA as indications for AAPs (bipolar disorder and schizophrenia) or conditions that are commonly treated with AAPs, as suggested by clinicians (pyschotic disorder, paranoia, psychosis, tics/Tourette's syndrome, and dementia); the exclusion of these conditions was made to increase the likelihood that patients received AAPs for ADHD and not other indications for which AAPs are commonly used. In addition, patients were required to use the index drug for switching or augmentation purposes (i.e., patients were either switching from a previous stimulant to the index drug or augmenting a previous stimulant with the index drug). Switching was defined as patients who initiated the index drug within a period of 30 days before or after the last day with stimulant supply. Augmenting was defined as patients who had initiated the index drug after the stimulant and had at least 30 consecutive days overlapping supply between the stimulant and the index drug.

To control for observable selection bias, patients in the non-antipsychotic group (reference group) were matched oneto-one to patients in the AAP group using propensity score matching. The propensity score (i.e., the likelihood of receiving an AAP conditional on the covariates) was generated from an unconditional logistic regression. A greedy matching algorithm was utilized to perform the match. ${ }^{27}$ The patient characteristics considered in the propensity score model included age; gender; geographic region; year of therapy initiation; baseline stimulant class (amphetamine IR, amphetamine extendedrelease [XR], methylphenidate IR, and methylphenidate XR); duration of stimulant use before the index date; comorbidity profile (including accidental injury, adjustment reaction, anxiety disorders, asthma, conduct disorder, depression, epilepsy, insomnia, learning disability, neurological disorder, obsessive compulsive disorder, oppositional defiant disorder, and substance abuse), as well as all-cause and mental health-related medical care utilization (inpatient, emergency room [ER], outpatient visits) and pharmacy and medical costs (based on the exact amount paid by the third-party payer, after rebates) during the 6-month pre-index period. In addition, an exact match on whether the index drug was switched to or augmented was required. The precision of the match was assessed by graphically comparing the overlap between the estimated propensity score between matched and unmatched patients (lack of overlap would indicate the presence of extreme patients that were not well represented in both cohorts). ${ }^{28}$ To assess the degree of balance after the propensity score match, the baseline characteristics were compared between the two matched cohorts, using McNemar tests for categorical variables and Wilcoxon signed-rank tests for continuous variables.

\section{Outcomes}

All outcomes were measured during the 12-month period following the index date. Outcome categories included treatment patterns, health care utilization, and health care costs. 


\section{Comparative Treatment Patterns, Resource Utilization, and Costs in Stimulant-Treated Children with ADHD Who Require Subsequent Pharmacotherapy with Atypical Antipsychotics Versus Non-Antipsychotics}

Treatment patterns included discontinuation, switching, and augmentation. Discontinuation with the index therapy was defined as a gap in the usage of the index therapy greater than 30 days. The discontinuation date was defined as the last day of supply of the index medication preceding a gap in usage greater than 30 days. A switch was defined as the initiation of a new ADHD medication (either an AAP or a non-antipsychotic other than the index therapy or the current stimulant) within 30 days before or after the index therapy discontinuation date. Because patients who switch medication inherently both discontinue and initiate a novel therapy, all patients who switched were a subgroup of those who discontinued. Augmentation was defined as initiation of a new ADHD medication that had at least 30 days of supply overlap with the index therapy.

Health care utilization outcomes included three mutually exclusive categories: inpatient/hospital services, ER visits, and outpatient services. Inpatient claims and outpatient claims were used as provided in the database. Claims for ER services were separated from inpatient and outpatient claims based on the place of service, provider type, service type, and procedure group codes according to the algorithm provided in the database user guide. In each category, both all-cause utilization and mental health-related utilization were examined. Mental health-related utilization was defined as services associated with a primary diagnosis of a mental health condition (ICD9-CM: 290-319). Cause of urgent care utilization (i.e., inpatient and ER visits) was determined based on the primary diagnosis. The identified causes were further classified into different mental health-related causes according to groupings of psychiatric comorbidities from the Diagnostic and Statistical Manual of Mental Disorders, fourth edition (DSM-IV) ${ }^{29}$

Health care costs included medical costs (i.e., costs for hospitalizations, ER visits, and outpatient visits) and prescription drug costs (including the index drug). Both all-cause and mental health-related costs were evaluated. Mental health-related medical costs were defined as the costs of services associated with a primary diagnosis of a mental health condition (ICD9-CM: 290-319). Mental health-related drug costs were identified using the American Hospital Formulary Service (AHFS) therapeutic class codes and specific drug names for nonstimulants (AHFS does not have a specific category for nonstimulants). Cost analyses were conducted from a third-party payer's perspective (i.e., costs were defined as the amount paid by third-party payers, not including out-of-pocket costs paid by patients, such as deductibles, coinsurance, and copayments). All costs were accrued during the 12-month period immediately following the index date and inflated to 2010 U.S. dollars using the medical component of the Consumer Price Index. ${ }^{30}$

\section{Statistical Analysis of Outcomes}

Patient baseline characteristics, including age, gender, region, selected comorbidities, ADHD treatment, medical utilization, and costs during the 6 months pre-index date, were compared between patients in the AAP cohort and the non-antipsychotic cohort before propensity score matching. Specifically, Pearson chi-square tests were used to compare categorical variables, and Wilcoxon rank-sum tests were used to compare continuous variables in the pre-matched sample.

Rates of index drug discontinuation, switching, and augmentation were estimated using the Kaplan-Meier (KM) survival estimator. The hazard ratios (HR) of discontinuation, switching, and augmentation between the AAP cohort and the non-antipsychotic cohort were estimated using Cox proportional hazard models. For the switching and augmentation analyses, patients were considered censored 30 days after their discontinuation date. Rates of all-cause and mental healthrelated utilizations (i.e., hospitalizations, ER visits, and outpatient visits) as well as rates of hospitalizations and ER visits related to specific causes were compared between subjects in the two matched cohorts using McNemar's test. Event rate ratios (ERR) for all-cause and mental health-related utilization between the AAP cohort and the non-antipsychotic cohort were estimated using Poisson regressions. Health care costs are typically not normally distributed and highly skewed to the right; therefore, they were compared between the two matched cohorts using nonparametric Wilcoxon signed-rank tests.

All analyses were performed on an intent-to-treat basis (i.e., patients were grouped by their index therapy) using SAS Version 9.2 (SAS Institute, Cary, NC). Statistical significance was evaluated at the 0.05 significance level (two-sided).

\section{Results}

A total of 441,673 children with at least one diagnosis of ADHD were identified in the Thomson Reuters MarketScan Commercial Claims \& Encounters database between January 1, 2005, and December 31, 2009 (Figure 1). Of these children, 271,165 (61.4\%) had at least one pharmacy claim for a stimulant medication. Among them, 22,622 (8.3\% of all pharmacologically treated ADHD patients) had at least one pharmacy claim for an AAP medication after the initial stimulant, and 84,558 (31.2\% of all pharmacologically treated ADHD patients) had at least one pharmacy claim for a non-antipsychotic medication (and no claims for an AAP) after the initial stimulant. Of the 22,622 patients identified to have used AAPs, 15,664 (69.2\%) patients did not have psychiatric diagnosis for which AAPs were FDA indicated or commonly used. Among the 84,558 patients using non-antipsychotics after a stimulant, 81,397 (96\%) did not have such psychiatric diagnoses. A total of 2,127 children in the AAP cohort and 16,508 children in the non-antipsychotic cohort met all the study inclusion criteria.

\section{Baseline Characteristics}

Before matching, there were significant differences in the patient demographics and other baseline characteristics 


\section{Comparative Treatment Patterns, Resource Utilization, and Costs in Stimulant-Treated Children with ADHD Who Require Subsequent Pharmacotherapy with Atypical Antipsychotics Versus Non-Antipsychotics}

\section{FIGURE 1 Sample Selection Flowchart (Child Patients: $6 \leq$ Age $<13$ )}

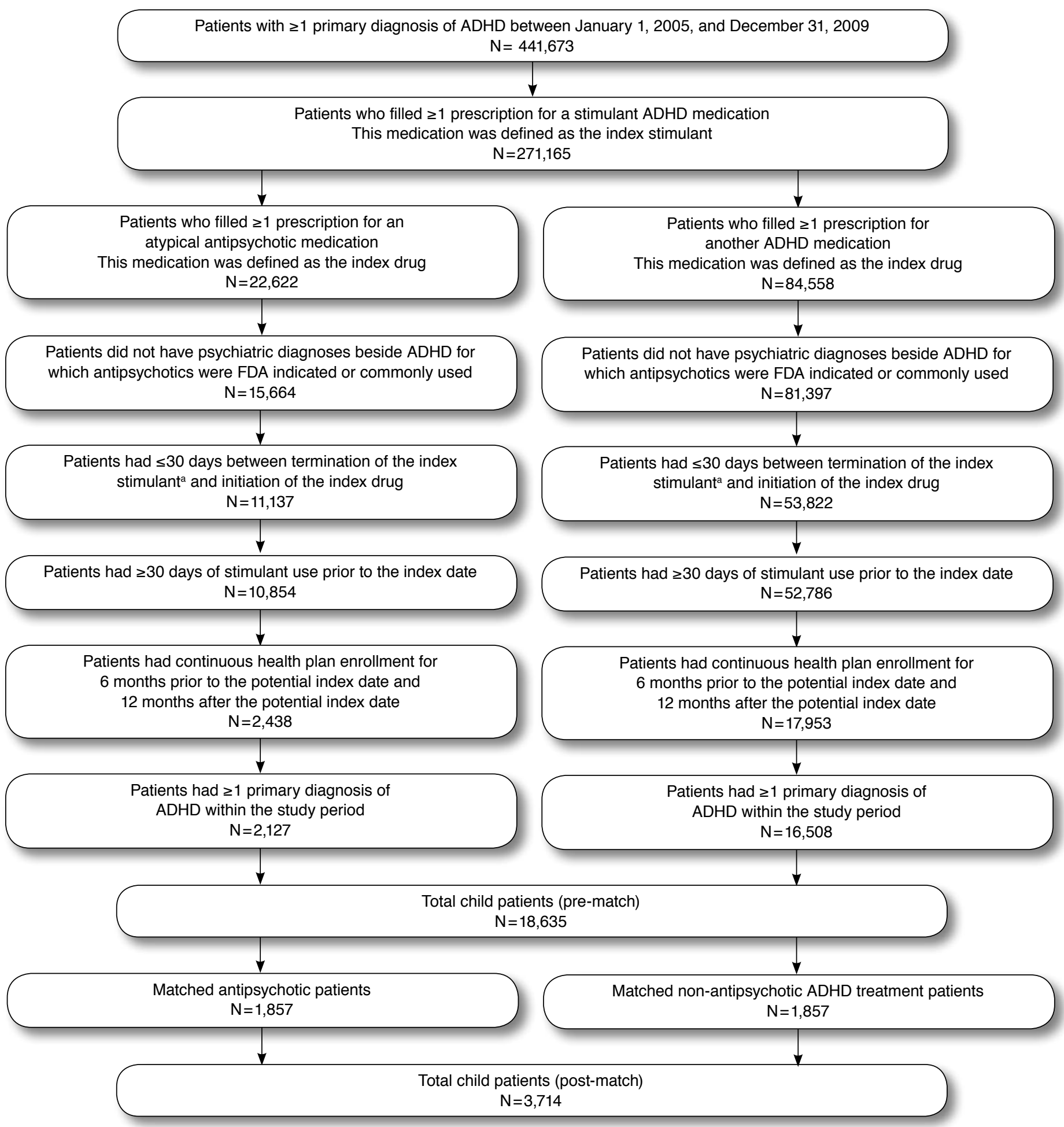

aTermination of the index stimulant was defined as discontinuation of the index stimulant. Termination of the index stimulant did not occur in all patients. ADHD = attention-deficit/hyperactivity disorder; FDA = U.S. Food and Drug Administration. 


\section{Comparative Treatment Patterns, Resource Utilization, and Costs in Stimulant-Treated Children with ADHD Who Require Subsequent Pharmacotherapy with Atypical Antipsychotics Versus Non-Antipsychotics}

TABLE 1 Comparison of Baseline Demographic Characteristics and Comorbidities: Atypical Antipsychotic and Non-Antipsychotic Cohorts (Pre- and Post-Matched)

\begin{tabular}{|c|c|c|c|c|c|c|c|}
\hline \multirow[b]{2}{*}{ Baseline Characteristics } & \multicolumn{4}{|c|}{ Pre-Match Sample } & \multicolumn{3}{|c|}{ Post-Match Sample } \\
\hline & \multicolumn{2}{|c|}{$\begin{array}{c}\text { Atypical } \\
\text { Antipsychotic } \\
\text { Patients } \\
\mathrm{N}=2,127 \\
\end{array}$} & $\begin{array}{c}\text { Non- } \\
\text { Antipsychotic } \\
\text { Patients } \\
\mathrm{N}=16,508 \\
\end{array}$ & \multirow[t]{2}{*}{$P$ Value $^{\mathrm{a}}$} & $\begin{array}{c}\text { Atypical } \\
\text { Antipsychotic } \\
\text { Users } \\
\mathrm{N}=1,857 \\
\end{array}$ & $\begin{array}{c}\text { Non- } \\
\text { Antipsychotic } \\
\text { Users } \\
\mathrm{N}=1,857 \\
\end{array}$ & \multirow[t]{2}{*}{$P$ Value $^{\mathrm{b}}$} \\
\hline \multicolumn{6}{|l|}{ Demographic characteristics } & & \\
\hline Age, mean \pm SD & \multicolumn{2}{|c|}{$9.11 \pm 1.97$} & $8.89 \pm 1.87$ & $<0.001$ & $9.0 \pm 2.0$ & $9.0 \pm 1.9$ & 0.904 \\
\hline Female, n (\%) & 458 & $(21.5)$ & $4,510 \quad(27.3)$ & $<0.001$ & $417 \quad(22.5)$ & $417 \quad(22.5)$ & 1.000 \\
\hline \multicolumn{8}{|l|}{ Region of residence, $\mathbf{n}(\%)$} \\
\hline Northeast & 215 & $(10.1)$ & 1,430 & 0.027 & $(9.5)$ & (9.7) & 0.869 \\
\hline Midwest & 735 & (34.6) & (29.9) & $<0.001$ & (33.3) & $(34.2)$ & 0.518 \\
\hline South & 853 & $(40.1)$ & $(49.2)$ & $<0.001$ & $(41.5)$ & $(41.0)$ & 0.783 \\
\hline West & 310 & (14.6) & (11.7) & $<0.001$ & (15.0) & $(14.5)$ & 0.668 \\
\hline National (unknown) & 14 & $(0.7)$ & $(0.6)$ & 0.797 & $(0.7)$ & $(0.5)$ & 0.394 \\
\hline \multicolumn{8}{|l|}{ Index drug switching vs. augmenting, n (\%) } \\
\hline Switching & 388 & $(18.2)$ & 10,714 & $<0.001$ & $(19.3)$ & (19.3) & 1.000 \\
\hline Augmenting & 1,739 & $(81.8)$ & $(35.1)$ & $<0.001$ & 1,499 & $(80.7)$ & 1.000 \\
\hline \multicolumn{8}{|l|}{ Baseline pharmacological ADHD treatment } \\
\hline Number of stimulants, mean \pm SD & & \pm 0.34 & $1.75 \pm 0.48$ & $<0.001$ & $1.1 \pm 0.4$ & $1.1 \pm 0.3$ & 0.065 \\
\hline Duration of stimulants, mean \pm SD & 93.5 & \pm 60.28 & $99.10 \pm 56.39$ & $<0.001$ & $92.6 \pm 59.6$ & $96.4 \pm 60.4$ & 0.047 \\
\hline Baseline psychotherapy, n (\%) & & & & & & & \\
\hline Number of visits, mean \pm SD & & \pm 5.69 & $1.50 \pm 3.48$ & $<0.001$ & $3.3 \pm 4.9$ & $3.4 \pm 5.5$ & 0.719 \\
\hline Number of patients with visit, $\mathrm{n}(\%)$ & 1,346 & $(63.3)$ & (31.9) & $<0.001$ & 1,102 & (59.4) & 0.973 \\
\hline Baseline stimulant use, n (\%) & & & & & & & \\
\hline Methylphenidate immediate-release & 404 & $(19.0)$ & (19.5) & 0.593 & $(18.2)$ & $(20.0)$ & 0.172 \\
\hline Methylphenidate extended-release & 1,298 & $(61.0)$ & 12,909 & $<0.001$ & 1,134 & $(63.5)$ & 0.116 \\
\hline Amphetamine immediate-release & 236 & (11.1) & (11.0) & 0.889 & $(10.5)$ & $(10.3)$ & 0.875 \\
\hline Amphetamine extended-release & 783 & $(36.8)$ & (59.3) & $<0.001$ & $(37.6)$ & $(34.5)$ & 0.050 \\
\hline Comorbidity profile, $\mathrm{n}(\%)$ & & & & & & & \\
\hline Accidental injury & 331 & $(15.6)$ & $(12.8)$ & $<0.001$ & $(14.6)$ & (13.4) & 0.294 \\
\hline Adjustment reaction & 207 & $(9.7)$ & 1,003 & $<0.001$ & (9.4) & $(8.6)$ & 0.390 \\
\hline Anxiety disorder & 188 & $(8.8)$ & (3.8) & $<0.001$ & (7.6) & $(7.8)$ & 0.901 \\
\hline Asthma & 88 & $(4.1)$ & (5.0) & 0.080 & $(4.3)$ & $(4.8)$ & 0.475 \\
\hline Conduct disorder & 157 & $(7.4)$ & $(2.4)$ & $<0.001$ & $(5.7)$ & $(5.1)$ & 0.426 \\
\hline Depression & 159 & $(7.5)$ & $(2.3)$ & $<0.001$ & $(6.2)$ & $(5.2)$ & 0.178 \\
\hline Epilepsy & 36 & $(1.7)$ & $(0.7)$ & $<0.001$ & (1.3) & (1.1) & 0.456 \\
\hline Insomnia & 18 & $(0.8)$ & $(0.5)$ & 0.052 & $(0.8)$ & $(0.9)$ & 0.578 \\
\hline Learning disability & 46 & $(2.2)$ & (2.3) & 0.613 & $(2.1)$ & $(2.6)$ & 0.335 \\
\hline Neurological disorders & 92 & $(4.3)$ & $(2.8)$ & $<0.001$ & $(4.0)$ & $(4.5)$ & 0.519 \\
\hline Obsessive compulsive disorder & 45 & $(2.1)$ & $(0.5)$ & $<0.001$ & (1.6) & (1.3) & 0.586 \\
\hline Oppositional defiant disorder & 171 & $(8.0)$ & $(2.2)$ & $<0.001$ & $(5.8)$ & $(5.4)$ & 0.614 \\
\hline Substance abuse & 5 & $(0.2)$ & $(0.1)$ & 0.004 & $(0.1)$ & $(0.2)$ & 0.655 \\
\hline Non-comorbid ADHDc & 1,292 & $(60.7)$ & 13,180 & $<0.001$ & 1,209 & $(66.1)$ & 0.512 \\
\hline Inpatient/hospitalizations & & & & & & & \\
\hline Patients with at least one visit, $\mathrm{n}(\%)$ & & $(4.2)$ & $(0.6)$ & $<0.001$ & $(2.6)$ & $(1.8)$ & 0.118 \\
\hline Number of visits per patient, mean \pm SD & 0.05 & $=0.23$ & $0.01 \pm 0.09$ & $<0.001$ & $0.0 \pm 0.2$ & $0.0 \pm 0.1$ & 0.055 \\
\hline Cost per patient, mean \pm SD & 684.26 & $9,003.88$ & $64.27 \pm 1,087.88$ & $<0.001$ & $282.7 \pm 2,736.3$ & $191.9 \pm 1,848.2$ & 0.221 \\
\hline Emergency room & & & & & & & \\
\hline Patients with at least one visit, $\mathrm{n}(\%)$ & 290 & (13.6) & 1,617 & $<0.001$ & (11.9) & (11.9) & 1.000 \\
\hline Number of visits per patient, mean \pm SD & 0.18 & 0.53 & $0.12 \pm 0.41$ & $<0.001$ & $0.2 \pm 0.5$ & $0.1 \pm 0.5$ & 0.880 \\
\hline Cost per patient, mean \pm SD & 89.02 & 354.95 & $61.49 \pm 306.62$ & $<0.001$ & $75.0 \pm 328.5$ & $81.0 \pm 434.3$ & 0.640 \\
\hline Outpatient & & & & & & & \\
\hline Patients with at least one visit, $\mathrm{n}(\%)$ & 2,054 & $(96.6)$ & 15,927 & 0.837 & 1,787 & 1,772 & 0.222 \\
\hline Number of visits per patient, mean \pm SD & 7.72 & 7.60 & $5.32 \pm 5.91$ & $<0.001$ & $7.0 \pm 7.1$ & $7.0 \pm 7.1$ & 0.880 \\
\hline Cost per patient, mean \pm SD & $1,322.57$ & $=2,331.92$ & $870.00 \pm 2,107.42$ & $<0.001$ & $1,156.9 \pm 2,063.4$ & $1,106.9 \pm 1,892.8$ & 0.990 \\
\hline Drug use & & & & & & & \\
\hline Number of total prescriptions per patient, mean \pm SD & 9.44 & 6.54 & $6.45 \pm 4.67$ & $<0.001$ & $8.5 \pm 5.6$ & $8.6 \pm 6.3$ & 0.815 \\
\hline Cost per patient, mean \pm SD & $1,131.35$ & $1,394.79$ & $705.03 \pm 977.77$ & $<0.001$ & $999.6 \pm 927.3$ & $973.2 \pm 1,577.1$ & $<0.001$ \\
\hline Total cost per patient & $3,227.21$ & $9,651.44$ & $1,700.79 \pm 2,903.68$ & $<0.001$ & $2,514.2 \pm 3,990.8$ & $2,353.0 \pm 3,797.3$ & $<0.001$ \\
\hline $\begin{array}{l}\text { efined as ADHD with the absence of the following dia } \\
\text { omnia, learning disability, neurological disorders, obs }\end{array}$ & riabl & $\begin{array}{l}\text { hi-squal } \\
\text { McNem } \\
\text { ent reac } \\
\text { je disord }\end{array}$ & positional defia & e & $\begin{array}{l}\text { us variables. } \\
\text { ous variables. } \\
\text { depression, ep } \\
\text { bstance abuse. }\end{array}$ & & \\
\hline
\end{tabular}




\section{FIGURE 2a Kaplan-Meier Analysis of Persistence}

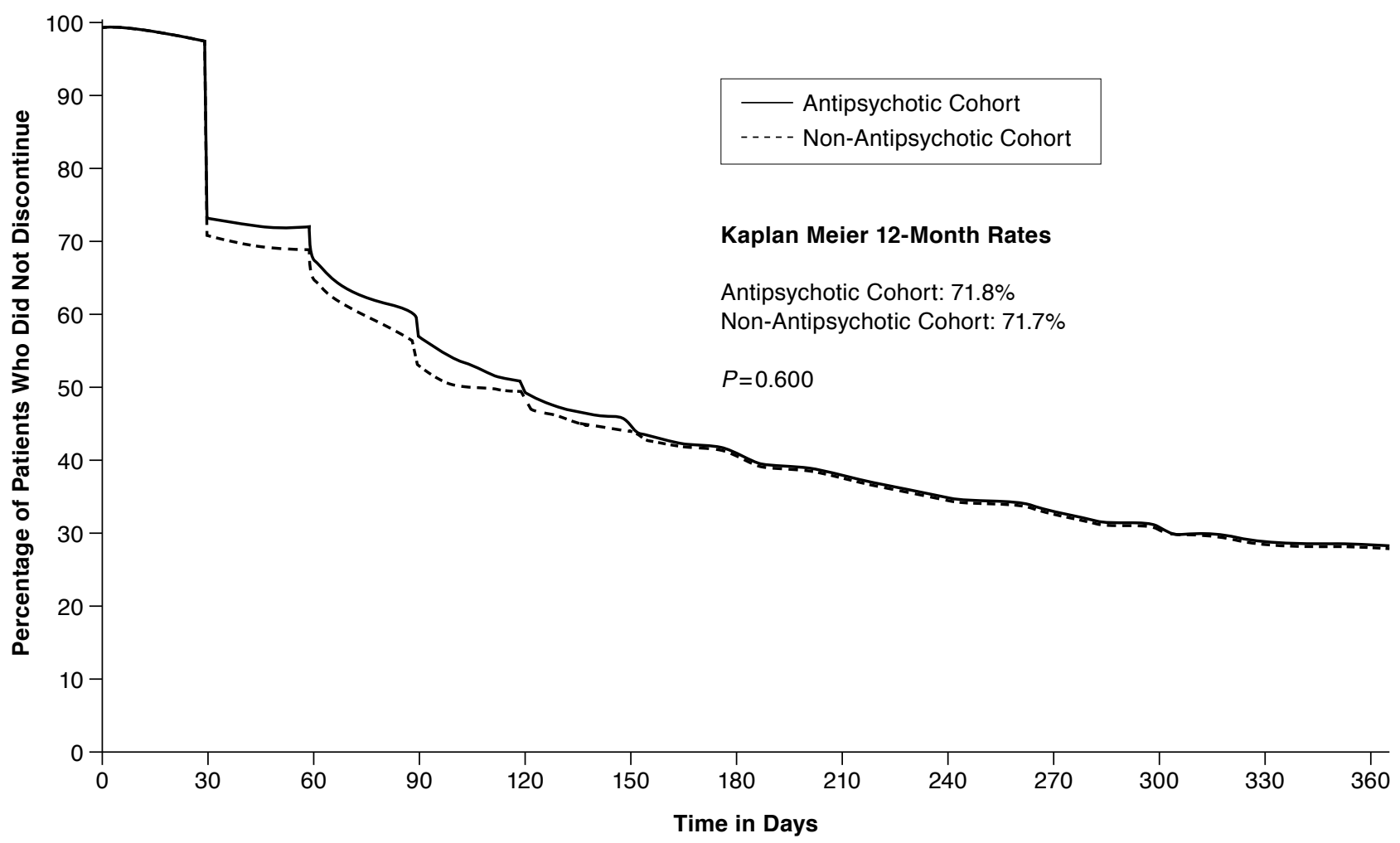

between the two cohorts (Table 1). During the baseline period, patients in the AAP cohort used fewer stimulants; the mean number of distinct stimulants used during the baseline period was 1.11 for the AAP cohort compared with 1.75 for the non-antipsychotic cohort. Moreover, patients in the AAP cohort were significantly less likely to use an extended-release methylphenidate $(61.0 \%$ vs. $78.2 \%)$ or an extended-release amphetamine (36.8\% vs. $59.3 \%$ ) compared with those in the non-antipsychotic cohort (all $P<0.001$ ). Patients in the AAP cohort were significantly more likely to have augmented with the index therapy rather than to have switched to the index therapy ( $81.8 \%$ vs. $35.1 \%)$ and to have had psychotherapy (63.3\% vs. $31.9 \%$ ), as well as anxiety disorder ( $8.8 \%$ vs. $3.8 \%)$, conduct disorder (7.4\% vs. $2.4 \%)$, depression ( $7.5 \%$ vs. $2.3 \%)$, and oppositional defiant disorder ( $8.0 \%$ vs. $2.2 \%$; all $P<0.001)$. Patients in the AAP cohort also had significantly more all-cause hospitalizations (0.05 vs. 0.01), ER visits (0.18 vs. 0.12) and outpatient visits (7.72 vs. 5.32), and higher all-cause costs due to hospitalization (\$684 vs. \$64), ER visits (\$89 vs. \$61), and outpatient visits ( $\$ 1,323$ vs. $\$ 870$; all $P<0.001$ ). With regards to mental health-related utilization and costs at baseline, AAP patients also had significantly more hospitalizations (0.04 vs. $<0.01$ ), ER visits (0.04 vs. 0.01), and outpatient visits (5.13 vs. 2.92) and had correspondingly higher hospitalization (\$308 vs.
\$21), ER (\$21 vs. \$6), and outpatient (\$701 vs. \$353) costs than non-antipsychotic patients (all $P<0.001$ ).

After propensity score matching, a total of 1,857 children (358 switchers and 1,499 augmenters) were included in each of the matched cohorts. The propensity scores for the non-antipsychotic cohort were found to have adequate overlap with the scores in the AAP cohort, indicative of the cohort's inclusion of a diverse group of patients without major outliers. The matched cohorts were well balanced, since all baseline demographics and almost all resource utilization characteristics were no longer significantly different (the matched AAP cohort were more likely to have used amphetamine XR $[P=0.050]$ and had a shorter duration of baseline stimulant use $[P=0.047]$, higher pharmacy costs $[P<0.001]$, and lower total costs $[P<0.001]$; Table 1)

Risperidone was the index drug for the majority of children in the matched AAP cohort (66.9\%), followed by aripiprazole (14.6\%), quetiapine (13.5\%), olanzapine (3.4\%), ziprasidone (1.3\%), paliperidone $(0.2 \%)$, and clozapine $(0.1 \%)$. These frequencies were very similar to what was observed in the pre-matched sample. In the pre-matched non-antipsychotic cohort, stimulants were the index drug for $70.4 \%$ of patients. However, in the post-matched sample, atomoxetine was the most common index drug (42.4\%), followed by clonidine IR 


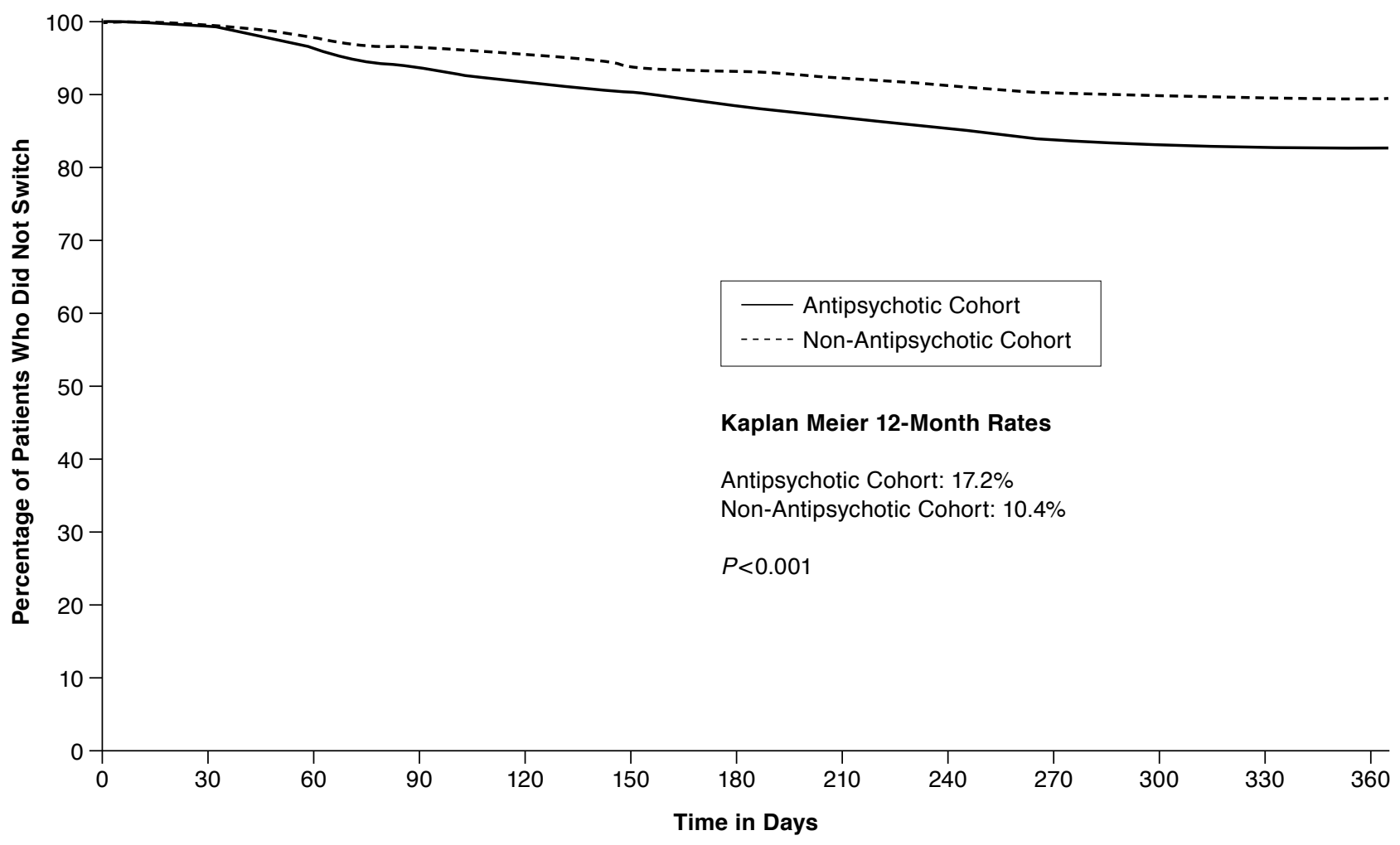

(35.6\%), guanfacine IR (11.3\%), and other stimulants (10.7\%). The difference before and after matching was in large part due to the fact that compared with stimulants, nonstimulants were more likely to be used as augmenting drugs. In the AAP cohort, $81.8 \%$ of stimulant users augmented with an AAP. Therefore, after exactly matching the index drug as an augmenting drug versus a switched-to drug, the distribution of the index therapy in the non-antipsychotic cohort changed substantially compared with the pre-matched distribution.

\section{Treatment Patterns}

The 12-month KM rate of discontinuation was similar between the AAP cohort and non-antipsychotic cohort $(71.8 \%$ vs. $71.7 \%$; $P=0.600$; Figure $2 \mathrm{a}$ ). The 12 -month KM rate of switching from the index drug to another ADHD medication was significantly higher in the AAP cohort compared with the nonantipsychotic cohort (17.2\% vs. 10.4\%; P<0.001; Figure 2b). The 12-month KM rate of augmentation was also significantly higher $(43.4 \%$ vs. $22.4 \% ; P<0.001)$ in the AAP cohort (Figure 2c). Overall, AAP patients were more likely to switch from the index drug $(\mathrm{HR}=1.75 ; P<0.001)$ and to augment the index drug $(\mathrm{HR}=2.62 ; P<0.001)$ compared with non-antipsychotic patients.

\section{Health Care Utilization}

During the 12-month study period, 84 (4.5\%) patients in the AAP cohort had at least one hospitalization, compared with $34(1.8 \%)$ patients in the non-antipsychotic cohort $(P<0.001$; Table 2). AAP patients had an average of 0.08 hospitalizations compared with 0.03 hospitalizations for the non-antipsychotic patients $(E R R=2.61 ; P<0.001)$. The majority of hospitalizations in both cohorts were related to mental health conditions (3.4\% vs. $1.1 \% ; P<0.001$ ). AAP patients had an average of 0.05 mental health-related hospitalizations compared with 0.02 mental health-related hospitalizations for the non-antipsychotic patients (ERR $=3.61 ; P<0.001)$. Mood disorders were the most common reason for hospitalizations among AAP patients and occurred significantly more frequently than in non-antipsychotic patients ( $1.8 \%$ vs. $0.2 \% ; P<0.001)$. The proportion of patients with at least one hospitalization attributed to ADHD was not significantly different between the two groups $(0.8 \%$ vs. $0.4 \% ; P=0.127$ ).

Rates of ER visits were also higher in the AAP cohort compared with the non-antipsychotic cohort $(23.8 \%$ vs. $18.5 \%$ at 12 months; $P<0.001$ ). AAP patients had an average of 0.34 ER visits during the 12 -month post-index period compared with $0.25 \mathrm{ER}$ visits for the non-antipsychotic patients $(E R R=1.33 ; P<0.001)$. The leading cause of ER visits in both 


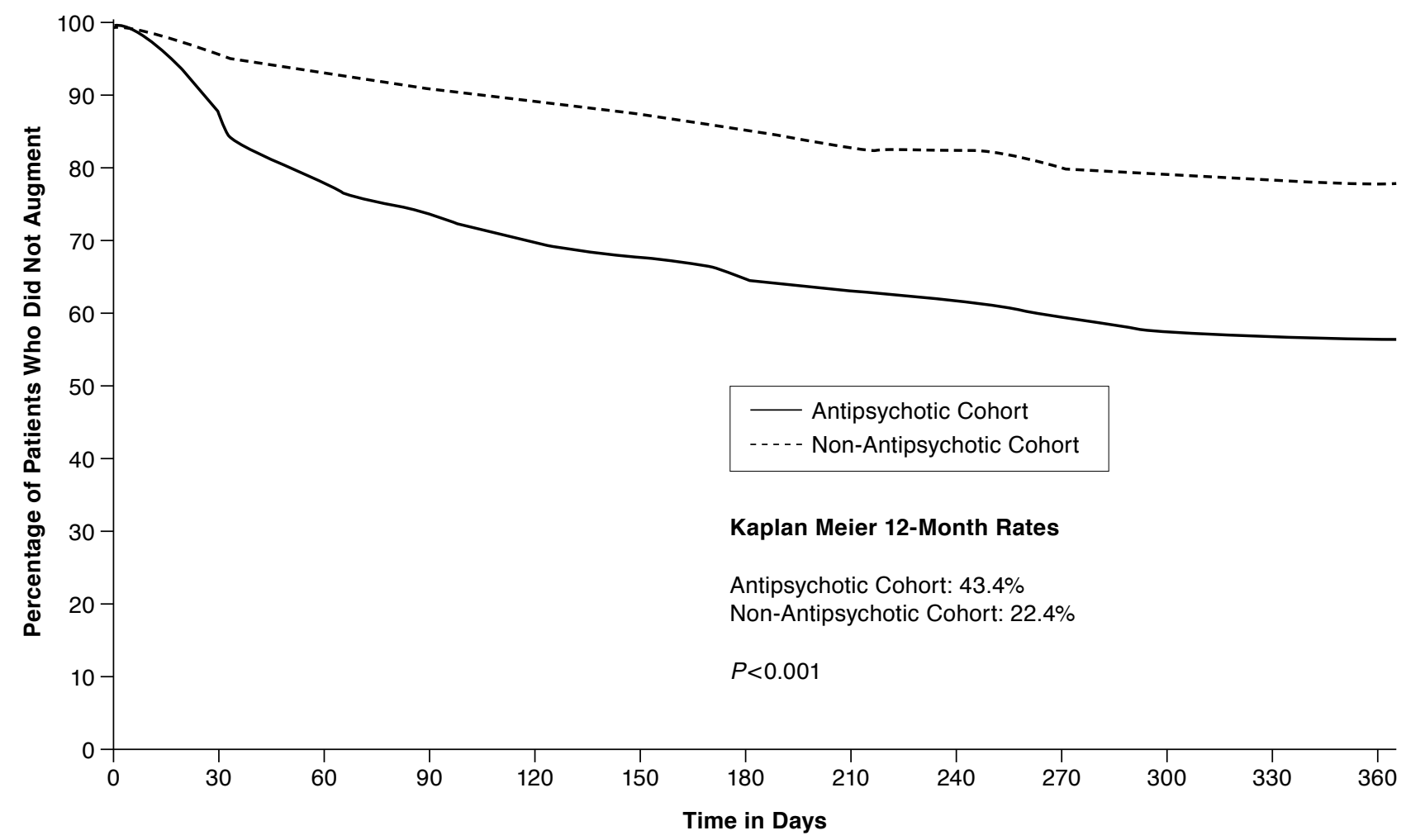

TABLE 2 Health Care Utilization: Atypical Antipsychotic and Non-Antipsychotic Cohorts (Among Matched Patients)

\begin{tabular}{|c|c|c|c|c|c|c|}
\hline \multirow{2}{*}{$\begin{array}{l}\text { Outcome Measures } \\
\text { Total health care }\end{array}$} & \multicolumn{2}{|c|}{$\begin{array}{l}\text { Atypical Antipsychotic Users } \\
\qquad \mathrm{N}=1,857\end{array}$} & \multicolumn{2}{|c|}{$\begin{array}{c}\text { Non-Antipsychotic Users } \\
\mathrm{N}=1,857\end{array}$} & \multirow{2}{*}{$\begin{array}{c}\text { Event Rate Ratios } \\
\text { ERR }(95 \% \mathrm{CI})\end{array}$} & \multirow[b]{2}{*}{$P$ Value $^{\mathrm{a}}$} \\
\hline & $\begin{array}{c}\text { Patients with Event } \\
\text { N (\%) }\end{array}$ & \multirow{2}{*}{$\frac{\text { Events per Patient }}{0.079}$} & $\begin{array}{c}\text { Patients with Event } \\
\text { N (\%) }\end{array}$ & \multirow{2}{*}{$\frac{\text { Events per Patient }}{0.030}$} & & \\
\hline Hospitalizations & $(4.52)$ & & $(1.83)$ & & $2.61 \quad(1.92-3.55)$ & $<0.001$ \\
\hline Emergency room visits & $441 \quad(23.75)$ & 0.339 & $344 \quad(18.52)$ & 0.254 & $1.33 \quad(1.18-1.50)$ & $<0.001$ \\
\hline Outpatient visits & $1,847 \quad(99.46)$ & 14.099 & $1,843 \quad(99.25)$ & 12.714 & $1.11 \quad(1.09-1.13)$ & $<0.001$ \\
\hline \multicolumn{7}{|l|}{ Mental health-related } \\
\hline Hospitalizations & (3.39) & 0.052 & $(1.08)$ & 0.017 & $3.13 \quad(2.09-4.69)$ & $<0.001$ \\
\hline Emergency room visits & $(4.85)$ & 0.054 & $(2.75)$ & 0.030 & $1.82 \quad(1.31-2.53)$ & $<0.001$ \\
\hline Outpatient visits & $1,769 \quad(95.26)$ & 9.304 & $1,755 \quad(94.51)$ & 7.689 & $1.21 \quad(1.18-1.24)$ & $<0.001$ \\
\hline
\end{tabular}

cohorts was accidents/injuries with a significantly higher rate in AAP patients (12.1\% vs. 9.6\%; $P=0.017)$. AAP patients also had significantly higher rates of ER visits related to mental health conditions ( $4.8 \%$ vs. $2.8 \%$; $P<0.001$ ), with an average of 0.05 mental health-related ER visits compared with 0.03 mental health-related ER visits for non-antipsychotic patients
(ERR $=1.82 ; P<0.001)$. The proportion of patients with at least one ER visit attributed to ADHD was not statistically different between the two cohorts ( $0.5 \%$ vs. $0.2 \% ; P=0.083)$.

Almost all patients had at least one outpatient visit in the AAP and non-antipsychotic cohorts (99.5\% vs. 99.3\%; $P=0.414)$ and at least one outpatient visit related to mental 


\section{Comparative Treatment Patterns, Resource Utilization, and Costs in Stimulant-Treated Children with ADHD Who Require Subsequent Pharmacotherapy with Atypical Antipsychotics Versus Non-Antipsychotics}

\section{TABLE 3 Health Care Costs: Atypical Antipsychotic and Non-Antipsychotic Cohorts}

(Among Matched Patients)

\begin{tabular}{|c|c|c|c|c|c|c|c|}
\hline \multirow{3}{*}{$\frac{\text { Outcome Measures }}{\text { Total health care costs }}$} & \multirow{2}{*}{\multicolumn{2}{|c|}{$\begin{array}{c}\text { Atypical Antipsychotic Users } \\
\mathrm{N}=1,857 \\
\text { Annual Cost }[\mathrm{A}] \\
\text { Mean [Median] }\end{array}$}} & \multirow{2}{*}{\multicolumn{2}{|c|}{$\begin{array}{c}\text { Non-Antipsychotic Users } \\
\mathrm{N}=1,857 \\
\text { Annual Cost [B] } \\
\text { Mean [Median] }\end{array}$}} & \multirow{2}{*}{\multicolumn{2}{|c|}{$\begin{array}{c}\text { Cost Difference } \\
{[\mathrm{A}] \text { - [B] }} \\
\text { Mean [Median] }\end{array}$}} & \multirow{3}{*}{$\frac{P \text { Value }}{<0.001}$} \\
\hline & & & & & & & \\
\hline & $6,934 \pm 8,695$ & {$[5,154]$} & $4,748 \pm 5,852$ & {$[3,394]$} & 2,186 & {$[1,760]$} & \\
\hline Total medical costs & $3,090 \pm 7,582$ & {$[1,394]$} & $2,238 \pm 4,515$ & {$[1,109]$} & 852 & [286] & $<0.001$ \\
\hline Inpatient & $647 \pm 5,740$ & [0] & $202 \pm 1,893$ & [0] & 445 & {$[0]$} & $<0.001$ \\
\hline Outpatient & $2,251 \pm 4,059$ & {$[1,189]$} & $1,903 \pm 3,436$ & {$[1,019]$} & 348 & {$[170]$} & $<0.001$ \\
\hline Emergency room & $192 \pm 577$ & {$[0]$} & $134 \pm 501$ & {$[0]$} & 59 & {$[0]$} & $<0.001$ \\
\hline Total drug cost & $3,844 \pm 2,903$ & {$[3,182]$} & $2,509 \pm 3,092$ & {$[1,892]$} & 1,334 & {$[1,290]$} & $<0.001$ \\
\hline Index drug & $1,587 \pm 1,623$ & {$[1,140]$} & $596 \pm 846$ & [139] & 991 & {$[1,001]$} & $<0.001$ \\
\hline Total mental health-related costs & $5,057 \pm 5,495$ & {$[4,026]$} & $2,859 \pm 2,396$ & {$[2,314]$} & 2,199 & {$[1,712]$} & $<0.001$ \\
\hline Medical & $1,555 \pm 4,708$ & [586] & $964 \pm 1,912$ & [434] & 592 & [152] & $<0.001$ \\
\hline Inpatient & $384 \pm 3,746$ & [0] & $80 \pm 1,080$ & [0] & 304 & [0] & $<0.001$ \\
\hline Emergency room & $28 \pm 185$ & {$[0]$} & $16 \pm 155$ & [0] & 12 & [0] & $<0.001$ \\
\hline Outpatient & $1,144 \pm 2,704$ & [554] & $867 \pm 1,526$ & [426] & 276 & [128] & $<0.001$ \\
\hline Drug & $3,502 \pm 2,358$ & {$[2,967]$} & $1,895 \pm 1,259$ & {$[1,631]$} & 1,607 & {$[1,336]$} & $<0.001$ \\
\hline
\end{tabular}

health (95.3\% vs. $94.5 \%$; $P=0.302)$. However, patients in the AAP cohort had on average significantly more outpatient visits (14.1 per year vs. 12.7 per year; ERR $=1.11 ; P<0.001)$ and significantly more outpatient visits related to mental health $(9.30$ per year vs. 7.69 per year; ERR=1.21; $P=0.001$ ).

\section{Health Care Costs}

Patients in the AAP cohort had higher health care costs during the 12-month post-index period compared with non-antipsychotic medication patients (Table 3 ). The average annual total health care costs for AAP patients were $\$ 6,934$ versus $\$ 4,748$ for non-antipsychotic patients $(P<0.001)$. Total mental healthrelated costs were also higher among AAP patients $(\$ 5,057$ vs. $\$ 2,859 ; P<0.001$ ).

The majority of total health care costs were attributable to pharmacy costs. The average 12-month drug cost was higher for AAP patients ( $\$ 3,844$ vs. $\$ 2,509 ; P<0.001)$. Mental healthrelated drug costs were also higher among AAP patients $(\$ 3,502$ vs. $\$ 1,895 ; P<0.001)$. In addition, patients in the AAP cohort also had higher costs for the index drug than patients in the non-antipsychotic cohort ( $\$ 1,587$ vs. $\$ 596 ; P<0.001)$.

The total all-cause mean medical costs were significantly higher for the AAP cohort $(\$ 3,090$ vs. $\$ 2,238 ; P<0.001)$. Outpatient costs constituted the largest component of the total all-cause medical costs for the AAP cohort and were significantly higher than the outpatient costs for the nonantipsychotic cohort $(\$ 2,251$ vs. $\$ 1,903 ; P<0.001)$. The AAP cohort also had higher all-cause inpatient costs ( $\$ 647$ vs. $\$ 202$; $P<0.001$ ), mental health-related inpatient costs ( $\$ 384$ vs. $\$ 80 ; P<0.001)$ as well as higher all-cause ER costs (\$192 vs.
$\$ 134 ; P<0.001$ ), mental health-related ER costs ( $\$ 28$ vs. $\$ 16$; $P<0.001)$, and mental health-related outpatient costs $(\$ 1,144$ vs. $\$ 867 ; P<0.001)$.

\section{Discussion}

Consistent with previous findings, ${ }^{14-16}$ this study found that $69 \%$ of the children with ADHD and receiving AAPs did not have a psychiatric diagnosis for which AAPs were FDA indicated or commonly used. These results highlight the prevalence of AAP use in this population and the need to better understand the economic and medical consequences of such use, especially given that clinical evidence supporting the treatment of ADHD with AAPs is limited. The current study employed a nationwide claims database to compare treatment patterns, health care utilization, and health care costs between children with ADHD who received stimulants and were subsequently treated with AAPs or non-antipsychotics. To our knowledge, this is the first study to examine the real-world treatment patterns and health care utilization and costs associated with AAPs as an off-label treatment for ADHD. This study showed that after controlling for measured baseline confounding factors between the two cohorts, AAP-treated patients had higher rates of switching and augmentation than the non-antipsychotic treated patients. Assuming switching and augmentation are associated with suboptimal treatment with the index therapy, ${ }^{31,32}$ these data potentially suggest that patients treated with AAPs are more likely to have suboptimal responses compared with those in the non-antipsychotic cohort. The findings are consistent with the current literature, which does not support the clinical use of AAPs for the treatment of ADHD. ${ }^{22-25}$ 


\section{Comparative Treatment Patterns, Resource Utilization, and Costs in Stimulant-Treated Children with ADHD Who Require Subsequent Pharmacotherapy with Atypical Antipsychotics Versus Non-Antipsychotics}

In addition, this study found that AAP-treated patients had higher rates of medical resource utilization (including outpatient, inpatient, and ER visits), as well as higher health care costs. The incremental total all-cause health care costs associated with AAP use were more than $\$ 2,000$ during the first 12 months of treatment. The high health care costs among AAPtreated patients were consistent across all cost components and for both all-cause and mental health-related services. The current study did not evaluate the reasons behind the increased resources use, which could be potentially related to the degree of ADHD symptom control, ${ }^{5,8}$ side effects of AAPs, ${ }^{33-35}$ or other reasons. Further research is needed to investigate the exact causes.

In recent years, several major treatment and formulary changes occurred, which could affect the management of ADHD and subsequently the associated costs of ADHD treatment. For example, the FDA approved the extended-release stimulant lisdexamfetamine (February 2007), as well as two nonstimulants, guanfacine XR (September 2009), and clonidine XR (October 2010) for the treatment of ADHD. Also within this time span, several generic medications entered the market, including generic formulations of risperidone as well as generic formulations of some stimulants.

The addition of new treatments and formulations as well as the shifting of prescribing patterns over time may influence the clinical and economic outcomes of ADHD patients. Given that our study found that the majority of health care costs were attributed to total drug costs $(55.4 \%$ and $52.8 \%$ of the total health care cost in the AAP and non-antipsychotic cohorts, respectively), a sensitivity analysis to estimate the impact of new drug entries on the study findings was conducted. This sensitivity analysis assumed that all patients who used branded risperidone (approximately 10\% of AAP patients) would switch to generic risperidone, and all patients who used the IR formulations of guanfacine and clonidine (approximately $47 \%$ of non-antipsychotic patients) would switch to the XR formulations. Branded risperidone costs were reduced to generic risperidone costs using a ratio between the branded and generic risperidone costs observed in the study sample. Observed costs for generic guanfacine IR and clonidine IR were replaced with the costs for brand guanfacine XR and clonidine XR, respectively, based on the average wholesale prices (AWP). Applying the generic risperidone and as well as brand guanfacine XR and clonidine XR adjustments lowered the total drug costs by $\$ 257$ in the non-antipsychotic cohort and raised total drug costs by $\$ 561$ in the AAP cohort. Nonetheless, AAP patients still had higher index drug costs $(\$ 1,129$ vs. $\$ 1,105 ; P<0.001)$, total drug costs $(\$ 3,587$ vs. $\$ 3,070 ; P<0.001)$, and total health care costs $(\$ 6,677$ vs. $\$ 5,308 ; P<0.001)$ compared with nonantipsychotic patients.
Given the limited clinical evidence of efficacy and safety of AAPs in the treatment of ADHD and the substantial economic burden associated with their use demonstrated by the current study, further investigation of treating ADHD children with AAPs is warranted. The findings from this study suggest that the off-label use of treatments not supported by adequate clinical and economic evidence may pose a substantial economic burden to payers. The need for such evidence is especially pronounced with regards to ADHD, where there are already several FDA-approved therapies, including both stimulants and nonstimulants with demonstrated effectiveness as monotherapy or adjunctive therapy. A retrospective drug utilization review (DUR) for off-label treatment of ADHD could help managed care organizations better understand the reasons AAPs are being prescribed and monitor patient outcomes. Such a DUR could potentially improve the quality of care for children with ADHD by ensuring AAPs are only used by patients who can benefit from the treatment, which may eventually reduce the economic burden associated with ADHD treatment.

\section{Limitations}

First, it cannot be conclusively determined whether AAPs were used specifically for the treatment of ADHD from the administrative claims data. To increase confidence in attributing medication usage to the treatment of ADHD, the following selection criteria were imposed: patients had to have continuously used a stimulant for at least 30 days prior to AAP initiation and had to have had no psychiatric diagnoses during the baseline and study periods for which AAPs are FDA-approved or often used. However, there is still a possibility that the AAPs were used for psychiatric disorders that were not included in our exclusion criteria or other psychiatric disorders that were not documented in the claims database. For example, if explosive aggression was a target AAP treatment, physicians may not be familiar with a diagnosis such as Intermittent Explosive Disorder or do not feel that chronically aggressive children meet the criteria given the intermittent label. Thus, the lack of appropriate diagnoses in the DSM-IV ${ }^{29}$ may lead to underdiagnosis in the claims database. It is also possible that psychiatric conditions are underdiagnosed in the claims data due to the stigma attached to a psychiatric diagnosis. On the other hand, the stringent criteria may have also eliminated users with ADHD and certain comorbid psychiatric disorders; thus, the results may be less generalizable to the entire ADHD population. It is also possible that AAPs were being used for other off-label indications, such as conduct disorders or oppositional defiant disorder. However, these comorbidities were included in the propensity score match, and there were no significant differences between the two cohorts in the frequency of these diagnoses. 


\section{Comparative Treatment Patterns, Resource Utilization, and Costs in Stimulant-Treated Children with ADHD Who Require Subsequent Pharmacotherapy with Atypical Antipsychotics Versus Non-Antipsychotics}

Second, although the patient cohorts were matched on available baseline variables, confounding due to differences in unobserved variables may remain. For example, although propensity score matching with selected comorbidities, as well as baseline health care utilization and costs, was used to control for baseline ADHD severity between AAP and non-antipsychotic cohorts, there may still be residual differences in disease severity between the two cohorts. Such unobserved confounding is a common limitation in observational studies.

Third, in order to create comparable cohorts, propensity score matching was used. Therefore, we cannot extend these results to the entire non-antipsychotic population that we did not match. The baseline characteristics of the unmatched non-antipsychotic patients tended to be less severe than the matched non-antipsychotic patients, and these unmatched patients may experience lower total health care costs. However, in the sense that these patients may not be candidates for AAPs, our study presents a valid comparison between AAP and non-antipsychotics in the population for which AAPs are a potential treatment.

Fourth, because the cost analysis only covered the first 12 months following the index date, this study cannot provide insight towards the long-term economic implications associated with AAP treatment. Also, the results may understate the true impact of treatment with AAPs, since many of the complications associated with AAPs are chronic conditions and the totality of their costs may not manifest within the 12-month study period. Future research is warranted to extend the comparison to a longer time horizon.

Finally, these results are only generalizable to the U.S. commercially insured population, so the study population may not be representative of all children with ADHD in the United States. For example, Medicaid patients may have different patient characteristics, disease severity, and treatment patterns compared with commercially insured patients. Further research in the Medicaid population may be warranted.

\section{Conclusions}

Compared with patients treated with stimulants who switched to or augmented with non-antipsychotics (atomoxetine, guanfacine IR, clonidine IR, or a stimulant of a different class than the initial stimulant), those who switched to or augmented with AAPs had significantly higher rates of switching and augmentation, greater medical resource utilization, and higher total health care costs. As off-label AAP use increases, these patients may present a greater economic burden to payers. Further outcomes research and/or drug utilization reviews may be warranted to fully evaluate clinical and economic outcomes in children diagnosed with ADHD who are receiving AAPs.

\section{Authors}

VANYA SIKIRICA, PharmD, MPH, is Director, Global Health Economics and Outcomes Research; PAUL HODGKINS, PhD, MSc, is Senior Director, Global Health Economics and Outcomes Research; M. HAIME ERDER, PhD, is Vice President, Global Health Economics and Outcomes Research and Epidemiology; RYAN DAMMERMAN, MD, PhD, is Director, Global Medical Affairs; and BRIGITTE ROBERTSON, MD, Senior Director, Global Clinical Development and Innovation, Shire Development LLC, Wayne, Pennsylvania. KEITH A. BETTS, PhD, is Associate; TOM SAMUELSON, BA, is Senior Analyst; JIPAN XIE, MD, PhD, is Manager; and ERIC Q. WU, PhD, is Managing Principal, Analysis Group, Inc., Boston, Massachusetts. STEVEN R. PLISZKA, MD, is Professor and Chief, Division of Child and Adolescent Psychiatry, Department of Psychiatry, The University of Texas Health Science Center at San Antonio, San Antonio, Texas.

AUTHOR CORRESPONDENCE: Vanja Sikirica, PharmD, MPH, Global Health Economics and Outcomes Research, Shire Development LLC, 725 Chesterbrook Blvd., Wayne, PA 19087. Tel: 484-595-8579; E-mail: vsikirica@shire.com.

\section{DISCLOSURES}

Funding for this research was provided by Shire Development LLC (SDLLC) Shire Pharmaceuticals LLC (SPLLC) is the parent company of SDLLC, which is a research and development organization. SPLLC is the manufacturer of lisdexamfetamine dimesylate, mixed salts of a single-entity amphetamine product, methylphenidate hydrochloride, and guanfacine extended-release for the treatment of ADHD. Vanja Sikirica, Paul Hodgkins, M. Haim Erder, Ryan Dammerman, and Brigitte Robertson are employees of SDLLC and stock/option owners of SPLLC. Keith A. Betts, Tom Samuelson, Jipan Xie, and Eric Q. Wu are employees of Analysis Group, Inc., which received funding from SDLLC for this study. Pliszka reports consulting work for SDLLC and has received grants from SDLLC and Ortho-McNeil-Janssen Pharmaceuticals, Inc. He has also provided expert testimony for Eli Lilly and Company. Wilson Joe from MedErgy provided editorial assistance in formatting, proofreading, and copy editing. This support was funded by SDLLC. Gina D'Angelo, PharmD, MBA, from SDLLC also reviewed and edited the manuscript for scientific accuracy.

Although the sponsor was involved in the design, collection, analysis, interpretation, and fact checking of information, the content of this manuscript, the ultimate interpretation, and the decision to submit it for publication in the Journal of Managed Care Pharmacy were made by all the authors independently. Preliminary results of this research were presented as a poster at the Academy of Managed Care Pharmacy 2011 Educational Conference in Atlanta, Georgia, October 19-21, 2011

Betts, Erder, Sikirica, and Pliszka were primarily responsible for concept and design. Samuelson, Xie, and Wu were primarily responsible for data collection. Pliszka, Dammerman, Betts, and Sikirica were primarily responsible for data interpretation. Sikirica, Pliszka, Betts, and Hodgkins were primarily responsible for writing the manuscript. Hodgkins, Robertson, Sikirica, and Pliszka were primarily responsible for revision of the manuscript. 


\section{Comparative Treatment Patterns, Resource Utilization, and Costs in Stimulant-Treated Children with ADHD Who Require Subsequent Pharmacotherapy with Atypical Antipsychotics Versus Non-Antipsychotics}

\section{REFERENCES}

1. Pastor PN, Reuben CA. Diagnosed attention deficit hyperactivity disorder and learning disability: United States, 2004-2006. Vital Health Stat 10. 2008;(237):1-14.

2. Centers for Disease Control and Prevention. Increasing prevalence of parent-reported attention-deficit/hyperactivity disorder among children-United States, 2003 and 2007. MMWR Morb Mortal Wkly Rep. 2010;59(44);1439-43.

3. Swensen AR, Birnbaum HG, Secnik K, et al. Attention-deficit/hyperactivity disorder: increased costs for patients and their families. J Am Acad Child Adolesc Psychiatry. 2003;42(12):1415-23.

4. Pelham WE, Foster EM, Robb JA, et al. The economic impact of attentiondeficit/hyperactivity disorder in children and adolescents. Ambul Pediatr. 2007;7(Suppl 1):S121-S131

5. Guevara J, Lozano P, Wickizer T, et al. Utilization and cost of health care services for children with attention-deficit/hyperactivity disorder. Pediatrics 2001;108(1):71-78

6. Swensen A, Birnbaum HG, Ben Hamadi R, et al. Incidence and costs of accidents among attention-deficit/hyperactivity disorder patients. J Adolesc Health. 2004;35(4):346.el-9.

7. Burd L, Klug MG, Coumbe MJ, et al. Children and adolescents with attention deficit-hyperactivity disorder: 1. Prevalence and cost of care.

J Child Neurol. 2003;18(8):555-61.

8. Leibson CL, Katusic SK, Barbaresi WJ, et al. Use and costs of medical care for children and adolescents with and without attention-deficit/hyperactivity disorder. JAMA. 2001;285(1):60-66

9. Mandell DS, Guevara JP, Rostain AL, Hadley TR. Economic grand rounds: medical expenditures among children with psychiatric disorders in a Medicaid population. Psychiatr Serv. 2003;54(4):465-67

10. Chan E, Zhan C, Homer CJ. Health care use and costs for children with attention-deficit/hyperactivity disorder: national estimates from the medical expenditure panel survey. Arch Pediatr Adolesc Med. 2002;156(5):504-11.

11. Harman JS, Childs GE, Kelleher KJ. Mental health care utilization and expenditures by children in foster care. Arch Pediatr Adolesc Med. 2000;154(11):1114-17.

12. Jensen PS, Garcia JA, Glied S, et al. Cost-effectiveness of ADHD treatments: findings from the multimodal treatment study of children with ADHD. Am J Psychiatry. 2005;162(9):1628-36.

13. Olfson M. New options in the pharmacological management of attention-deficit/hyperactivity disorder. Am J Manag Care. 2004;10(4 Suppl): S117-S124.

14. Kratochvil CJ. New ADHD treatment options on the horizon. Advanced Studies in Medicine. 2002;2(25):915-18.

15. Cooper WO, Arbogast PG, Ding H, Hickson GB, Fuchs DC, Ray WA Trends in prescribing of antipsychotic medications for US children. Ambul Pediatr. 2006;6(2):79-83. Available at: http://psychrights.org/articles/ KidAtypicalPrescribingTrends.pdf. Accessed November 27, 2012.

16. Pathak P, West D, Martin BC, Helm ME, Henderson C. Evidence-based use of second-generation antipsychotics in a state Medicaid pediatric population, 2001-2005. Psychiatr Serv. 2010;61(2):123-29.

17. Weiss M, Panagiotopoulos C, Giles L, et al. A naturalistic study of predictors and risks of atypical antipsychotic use in an attention-deficit/hyperactivity disorder clinic. J Child Adolesc Psychopharmacol. 2009;19(5):575-82.

18. Zuvekas SH. Prescription drugs and the changing patterns of treatment for mental disorders, 1996-2001. Health Aff (Millwood). 2005;24(1)195-205.

19. Alexander GC, Gallagher SA, Mascola A, Moloney RM, Stafford RS Increasing off-label use of antipsychotic medications in the United States, 1995-2008. Pharmacoepidemiol Drug Saf. 2011;20(2):177-84.
20. Maglione M, Ruelaz Maher A, Hu J. Off-label use of atypical antipsychotics: an update. Comparative Effectiveness Review No. 43. (Prepared by the Southern California/RAND Evidence-based Practice Center under Contract No. HHSA290-2007-10062-1.) AHRQ Publication No. 11-EHC087EF. Rockville, MD: Agency for Healthcare Research and Quality. September 2011. Available at: http://www.effectivehealthcare.ahrq.gov/search-forguides-reviews-and-reports/?pageaction=displayproduct $\&$ productid $=786$ Accessed November 27, 2012.

21. Findling RL, Steiner H, Weller EB. Use of antipsychotics in children and adolescents. J Clin Psychiatry. 2005;66(Suppl 7):S29-S40.

22. Armenteros JL, Lewis JE, Davalos M. Risperidone augmentation for treatment-resistant aggression in attention deficit/hyperactivity disorder: a placebo-controlled pilot study. J Am Acad Child Adolesc Psychiatry. 2007;46(5):558-65.

23. Correia Filho AG, Bodanese R, Silva TL, et al. Comparison of risperidone and methylphenidate for reducing ADHD symptoms in children and adolescents with moderate mental retardation. J Am Acad Child Adolesc Psychiatry. 2005;44(8):748-55.

24. Tramontina S, Zeni CP, Ketzer CR, Pheula GF, Narvaez J, Rohde LA. Aripiprazole in children and adolescents with bipolar disorder comorbid with attention-deficit/hyperactivity disorder: a pilot randomized clinical trial. J Clin Psychiatry. 2009;70(5):756-64

25. Zeni CP, Tramontina S, Ketzer CR, et al. Methylphenidate combined with aripiprazole in children and adolescents with bipolar disorder and attention-deficit/hyperactivity disorder: a randomized cossover trial. J Child Adolesc Psychopharmacol. 2009;19(5):553-61.

26. Kutcher S, Aman M, Brooks SJ, et al. International consensus statement on attention-deficit/hyperactivity disorder (ADHD) and disruptive behaviour disorders (DBDs): clinical implications and treatment practice suggestions. Eur Neuropsychopharmacol. 2004;14(1):11-28.

27. Bergstralh EJ, Kosanke JL, Jacobsen SJ. Software for optimal matching in observational studies. Epidemiology. 1996;7(3):331-32

28. Rosenbaum PR, Rubin DB. The central role of the propensity score in observational studies for causal effects. Biometrika. 1983;70(1):41-55. Available at: http://www.biostat.jhsph.edu/ jfeder/Journal_Club_files/ Biometrika\%201983\%20Rosenbaum.pdf. Accessed November 27, 2012

29. American Psychiatric Association. Diagnostic and Statistical Manual of Mental Disorders. 4th ed. Washington, DC: American Psychiatric Association; 2000.

30. U.S. Department of Labor, Bureau of Labor Statistics. Consumer Price Index. Available at: http://www.bls.gov/cpi/. Accessed November 27, 2012.

31. Christensen L, Sasané R, Hodgkins P, et al. Pharmacological treatment patterns among patients with attention-deficit/hyperactivity disorder: retrospective claims-based analysis of a managed care population. Curr Med Res Opin. 2010;26(4):977-89.

32. Erder MH, Signorovitch JE, Setyawan J, et al. Identifying patient subgroups who benefit most from a treatment: using administrative claims data to uncover treatment heterogeneity. J Med Econ. 2012;15(6):1078-87.

33. Maayan L, Correll CU. Weight gain and metabolic risks associated with antipsychotic medications in children and adolescents. J Child Adolesc Psychopharmacol. 2011;21(6):517-35.

34. Andrade SE, Lo JC, Roblin D, et al. Antipsychotic medication use among children and risk of diabetes mellitus. Pediatrics. 2011;128(6):1135-41.

35. Pringsheim T, Lam D, Ching H, Patten S. Metabolic and neurological complications of second-generation antipsychotic use in children: a systematic review and meta-analysis of randomized controlled trials. Drug Saf. 2011;34(8):651-68. 
Comparative Treatment Patterns, Resource Utilization, and Costs in Stimulant-Treated Children with ADHD Who Require Subsequent Pharmacotherapy with Atypical Antipsychotics Versus Non-Antipsychotics

\section{APPENDIX Codes for Atypical Antipsychotics}

and Non-Antipsychotics

(Stimulants + Nonstimulants)

\begin{tabular}{|c|c|c|}
\hline Drug Class & Drug & GPI Code ${ }^{a}$ \\
\hline \multirow{7}{*}{$\begin{array}{l}\text { Atypical } \\
\text { antipsychotics }\end{array}$} & Aripiprazole & 5925001500 \\
\hline & Clozapine & 5915202000 \\
\hline & Olanzapine & $\begin{array}{r}5915706000 \\
5915706010 \\
\end{array}$ \\
\hline & Paliperidone & 59070050 \\
\hline & Quetiapine & 5915307010 \\
\hline & Risperidone & 59070070 \\
\hline & Ziprasidone & 59400085 \\
\hline \multirow[t]{5}{*}{ Stimulants } & Dexmethylphenidate & 61400016 \\
\hline & Methylphenidate & 61400020 \\
\hline & Dextroamphetamine & 6110002010 \\
\hline & Lisdexamfetamine & 6110002510 \\
\hline & Amphetamine-Dextroamphetamine & 6110990210 \\
\hline \multirow[t]{3}{*}{ Nonstimulants } & Atomoxetine & 6135401510 \\
\hline & Clonidine IR & 362010101003 \\
\hline & Guanfacine IR & 3620102510 \\
\hline
\end{tabular}

aThe Medi-Span GPI is 14 characters in length; values less than 14 characters indicate that GPI codes beginning with this sequence were included. GPI = Generic Product Identifier; IR = immediate-release 